\title{
Nogo-A and Myelin-Associated Glycoprotein Mediate Neurite Growth Inhibition by Antagonistic Regulation of RhoA and Rac1
}

\author{
Barbara Niederöst, ${ }^{1}$ Thomas Oertle, ${ }^{2}$ Jens Fritsche, ${ }^{2}$ R. Anne McKinney, ${ }^{2}$ and Christine E. Bandtlow ${ }^{1}$ \\ 1/nstitute of Medical Chemistry and Biochemistry, Leopold-Franzens-University of Innsbruck, A-6020 Innsbruck, Austria, \\ and ${ }^{2}$ Brain Research Institute, University of Zurich and Swiss Federal Institute of Technology of Zurich, CH-8057 Zurich, \\ Switzerland
}

The adult mammalian CNS has a limited capacity for nerve regeneration and structural plasticity. The presence of gliaderived inhibitory factors myelin-associated glycoprotein (MAG) and Nogo-A have been suggested to provide a nonpermissive environment for elongating nerve fibers. In particular, Nogo-A, an integral membrane protein predominantly expressed by oligodendrocytes, has been demonstrated to impair neurite growth in vitro and in vivo. Structure function analysis revealed that Nogo-A protein contains at least two active domains, NiG and Nogo-66, with diverse effects on neurite outgrowth and cell spreading. We now provide evidence that these inhibitory domains mediate their effects via an antagonistic regulation of the small GTPases RhoA and Rac1, resulting in activation of RhoA and suppression of Rac1. By inactivating RhoA with C3 transferase or the downstream effector Rhokinase ROCK with Y27632, the inhibitory effects of both Nogo-A fragments and MAG on neurite outgrowth and oligodendrocyte-mediated growth cone collapse were abolished. Furthermore, we show that the recently cloned receptor for Nogo-66 and MAG, NgR, is not necessary for either NiG- or MAG-induced RhoA activation.

Key words: regeneration; neurite growth inhibitors; CNS myelin; Nogo-A; MAG; small GTPases; signal transduction
The molecular mechanisms by which axon regeneration is hampered in the adult mammalian CNS are poorly understood. The lack of growth-promoting molecules together with the presence of negative extracellular cues are thought to provide a nonpermissive environment for regrowing fibers. In particular, two components present in CNS myelin have been characterized as potent inhibitors of axonal growth: the myelin-associated glycoprotein (MAG) (Qiu et al., 2000) and Nogo-A, the largest transcript of the recently identified nogo gene (formerly called N I-220) (Chen et al., 2000; GrandPré et al., 2000; Prinjha et al., 2000). Depending on the developmental stage of the neuron, MAG and its soluble, cleaved form, dMAG, which is released in abundance from isolated or damaged myelin, can potently inhibit neurite outgrowth in vitro and are thought to be equally potent in vivo (Mukhopadhyay et al., 1994; DeBellard et al., 1996; Li et al., 1996; Schafer et al., 1996; Tang et al., 1997, 2001). Binding of MAG to sialic residues has been shown to be essential for neurite outgrowth inhibition (DeBellard et al., 1996), but the long sought neuronal receptor that transmits MAG signals remains elusive. Similarly, Nogo-A acts as a potent neurite growth inhibitor in vitro and represses axonal regeneration and structural plasticity in the adult mammalian CNS in vivo (Huber and Schwab, 2000). The inhibitory activity of Nogo-A resides in both the N-terminal part

\footnotetext{
Received April 8, 2002; revised Aug. 15, 2002; accepted Sept. 25, 2002.

This work was supported by Swiss National Science Foundation Grant 31-58398 and Austrian National Bank Project 9041 (C.E.B.). We thank Drs. M. Filbin for providing the MAG-CHO cells, A. Turnley for the cDNA coding for MAG-Fc fusion protein, A. Hall for the transferase C3-GST cDNA, M. Chao for the anti-p $75^{\text {NTR }}$ antibodies and the APtag5-vector, and M. Reindl for help with the statistical analysis.

Correspondence should be addressed to Christine E. Bandtlow, Institute of Medical Chemistry and Biochemistry, Division of Neurobiochemistry, LeopoldFranzens-University of Innsbruck, Fritz-Pregl-Straße 3, A-6020 Innsbruck, Austria. E-mail: christine.bandtlow@uibk.ac.at.

Copyright (C) 2002 Society for Neuroscience $0270-6474 / 02 / 2210368-09 \$ 15.00 / 0$
}

of the molecule, called NiG (Chen et al., 2000; Prinjha et al., 2000; Fournier et al., 2001), and the Nogo-66 domain, a stretch of 66 amino acids (aa) spanning the two putative transmembrane domains (GrandPré et al., 2000; Fournier et al., 2001). Moreover, $\mathrm{NiG}$ consists of several discrete regions that exhibit diverse inhibitory properties in vitro, the most potent being a 181 amino acid region (NiG- $\Delta 20)$ (Oertle et al., 2001) (T. Oertle and M. E. Schwab, unpublished observations).

The signaling mechanisms responsible for the transduction of the inhibitory properties of MAG and Nogo-A domains are not well understood. Recent evidence supports the notion that cytoskeletal components required for proper axonal pathfinding and the formation of axons and dendrites are differentially regulated by members of the Rho family, including RhoA, Rac1, and Cdc42 (Luo, 2000; Dickson, 2001). A complex cross-talk between Rho proteins seems to be crucial for this regulation (Dickson, 2001). Rho proteins serve as a molecular switch by cycling between an inactive GDP-bound state and an active GTP-bound state (Bishop and Hall, 2000). In their active state, these GTPases bind characteristic sets of effector proteins. The most important effector of RhoA in the growth cone is probably the serinethreonine kinase Rho-kinase ROCK (Bito et al., 2000). Genetic studies of ROCK function in growth cone guidance have not yet been reported, but in vitro experiments support the idea that ROCK is a negative regulator of growth cone motility (Bito et al., 2000).

In the present study, we examine the possible role of Rho proteins in mediating the neurite growth-inhibiting effects of MAG and the two functional domains of the Nogo-A molecule $\mathrm{NiG}$ and Nogo-66. Our results show that NiG, Nogo-66, and MAG oppositely regulate RhoA and Rac1, by enhancing RhoA and suppressing Rac1 activities in cerebellar granule cells. 


\section{MATERIALS AND METHODS}

Materials. Y27632, a specific inhibitor of the Rho-dependent serinethreonine kinase ROCK (Ishizaki et al., 2000), was a generous gift from Yoshitomi Pharmaceutical Industries (Saitama, Japan).

DNA constructs. The coding sequence for rat Nogo-66 (aa 1026-1091 of rat Nogo-A) was obtained by PCR using the primers 5'CTGGGATCCAGGATA TATAAGGGCGTG-3', containing a BamH1 site, and 5'-CGCTCGAGCTTCAGGGAAT C AACTAAATC-3', containing a $X h o I$ site. The restriction sites for directional cloning are underlined. The resulting PCR product was subcloned into the Bam HI/ XhoI sites of pGEX6-P (Amersham Biosciences, Uppsala, Sweden) for the production of glutathione $S$-transferase (GST) fusion proteins. The codon sequence for the rat Nogo-A-specific part describing aa 174-979 (rat $\mathrm{NiG}$ ) was obtained by partial digestion of rat Nogo-A-pET28 plasmid (pET28 was from Novagen, Madison, WI) with HincII. The resulting plasmid (rat NiR-G) was digested with $B a m \mathrm{HI} / B s a \mathrm{I}$, followed by Mung Bean nuclease treatment. All plasmids were sequenced to confirm that no errors were introduced.

Preparation of recombinant fusion proteins. Escherichia coli BL21/DE3 were transformed with the bacterial expression vectors and grown in 2xYT (Invitrogen, San Diego, CA) in the presence of $100 \mu \mathrm{g} / \mathrm{ml}$ ampicillin (GST-Nogo-66) or $30 \mu \mathrm{g} / \mathrm{ml}$ kanamycin (NiG). Expression of fusion proteins was induced by addition of $1 \mathrm{~mm}$ isopropyl-1-thio- $\beta$-Dgalactopyranoside (final concentration) (Roth, Reinach, Switzerland) to a $\log$ phase culture at $30^{\circ} \mathrm{C}$ for $3-4 \mathrm{hr}$. Cells were centrifuged at $10,000 \times$ $g$ for $10 \mathrm{~min}$ at $4^{\circ} \mathrm{C}$, and pellets were frozen at $-20^{\circ} \mathrm{C}$. For the purification of the highly expressed fusion proteins, cell pellets were resuspended with B-Per (Pierce, Rockford, IL), incubated on a shaker for 20 min, and centrifuged at $14,000 \times g$ for $20 \mathrm{~min}$ at $4^{\circ} \mathrm{C}$. Supernatants of GSTNogo-66 were purified with glutathione-Sepharose beads (Sigma, Deisenhofen, Germany) in a batch procedure according to the instructions of the manufacturer (Amersham Biosciences). For some experiments, the GST tag was removed by incubation of the solubilized rat GSTNogo-66 fusion protein with PreScission protease, followed by reversephase HPLC (RP-HPLC) (M. Zurini, Novartis, Basel, Switzerland). GST-C3 protein was cleaved from the GST by overnight incubation with thrombin (Sigma). Thrombin was removed by incubation with paraaminobenzamine-Sepharose (Amersham Biosciences), and the supernatant was concentrated to $1 \mathrm{mg} / \mathrm{ml}$ and dialyzed against $50 \mathrm{mM} \mathrm{NaCl}, \mathrm{pH}$ 7.5, $0.1 \mathrm{M} \mathrm{NaCl}$, and $5 \mathrm{mM} \mathrm{MgCl}_{2}$. Purity and complete removal of thrombin was confirmed by SDS-PAGE and silver staining. NiG expressed with pelB leader was obtained from the periplasmic space according to the Novagen protocol for periplasmic protein purification. Supernatants of pET28 constructs were purified using the $\mathrm{Co}^{2+}$-Talon Metal Affinity Resin (Clontech, Cambridge, UK) in a batch procedure. B-Per solubilized lysates were brought to nondenaturing conditions by increasingly substituting the buffer with sonication buffer during the resin-batch procedure. Proteins were eluted with $250 \mathrm{~mm}$ imidazole in 50 $\mathrm{mm} \mathrm{NaH}_{2} \mathrm{PO}_{4}, 20 \mathrm{~mm}$ Tris-HCl, and $100 \mathrm{~mm} \mathrm{NaCl}, \mathrm{pH}$ 8.0, on a gravity column (Bio-Rad, Hercules, CA). NiG was further purified by gel filtration on Superdex 200 HiLoad 16/60. Protein concentrations were determined with the BCA protein assay kit (Pierce) using bovine serum albumin (BSA) as a standard.

Preparation of alkaline phosphatase and Fc chimera. Rat NiG- $\Delta 20$ (aa $544-725$ of rat Nogo-A) and Nogo-66 (aa 1026-1091 of rat Nogo-A) were cloned into pAPtag5 vector. The PCR product using 5'-GAAGCTTACGTAATGGGTCGCGGATCCACAGG-3' and 5'-GTTGATTCCGGAAGAA AATAAGACAACTGGTTC- $3^{\prime}$ as a primer set for $\mathrm{NiG- \Delta 20}$ from NiG- $\Delta 20$-pET28 as a template was cloned into $S n a \mathrm{BI} / B s p \mathrm{EI}$ of pAPtag5, and the product using 5'-GACGAAGCTTACAGGATATATAAGGGCGTG-3' and 5'- GTTGATTCCGGACTTCAGGGAATCAACTAAATC-3' as primer sets for Nogo-66 was cloned into HindIII/ $B s p$ EI sites, respectively. Stable Chinese hamster ovary (CHO) cell lines secreting alkaline phosphatase (AP)-tagged NiG- $\Delta 20$ or Nogo-66 were derived from transient transfection of the above expression plasmids, followed by selection with $250 \mu \mathrm{g} / \mathrm{ml}$ zeocin (Invitrogen). Both cell lines were adapted to serum-free medium conditions and grown in a cell line chamber (Integra Biosciences, Baar, Switzerland) in CHO-S-SFM II medium (Invitrogen). Conditioned media from stably transfected cell lines were collected, and the protein production and integrity were confirmed by Western blot using anti-human AP antibodies. The dimerization and oligomerization of the fusion proteins was assessed by running samples in SDS-PAGE gel under reducing (sample buffer with $10 \%$ 2-mercaptoethanol) or nonreducing (sample buffer without 2-mercaptoethanol) conditions. For pull-down experiments, supernatant was concentrated 10-fold using Centriprep columns, and the concentrations of AP fusion proteins were assessed as described previously (Flanagan and Leder, 1990).

For the purification of MAG-Fc, conditioned media from transient transfected MAG-Fc-expressing human embryonic kidney 293 cells were collected, and buffer was exchanged into Protein A Sepharose binding buffer $\left(20 \mathrm{~mm} \mathrm{NaPO}_{4}, \mathrm{pH} 7.0\right)$. The protein was bound to Protein A Sepharose CL-4B resin (Amersham Biosciences) and eluted with $40 \mathrm{~mm}$ Na-citrate and $20 \mathrm{~mm} \mathrm{NaCl}, \mathrm{pH} 3.2$. The protein concentrations of MAG-Fc were estimated by comparing the intensity of Coomassie blue stain in SDS-PAGE gel using BSA as standard.

Neurite outgrowth assays. Cerebellar granule cells from postnatal day 7 (P7) rat pups were dissociated by combined trituration and trypsinization as described previously (Hatten, 1985). Cells were plated on four-well tissue culture dishes (Greiner Bio-One, Longwood, FL) coated with 10 $\mu \mathrm{g} / \mathrm{ml}$ poly-L-lysine (Sigma) and recombinant NiG and Nogo-66-GST fusion proteins. Cells were grown overnight in chemically defined Neurobasal medium (Invitrogen) supplemented with B27 (Invitrogen) and $0.2 \mathrm{~mm}$ glutamine, $100 \mathrm{U} / \mathrm{ml}$ penicillin, and $0.1 \mathrm{mg} / \mathrm{ml}$ streptomycin. Where indicated, Y27632 $(15 \mu \mathrm{M})$ was added to the cultures. For introduction of C3 transferase, we used a protocol modified from Borasio et al. (1989). Dissociated cerebellar granule cells were triturated in the presence of C3 transferase at $20 \mu \mathrm{g} / \mathrm{ml}$ together with $1 \mathrm{mg} / \mathrm{ml} 10,000 \mathrm{kDa}$ dextran-fluorescein (Molecular Probes, Eugene, OR) as an identification marker for cells that have taken up the fusion protein. More than $90 \%$ of the cells were labeled by this method. Granule cells were washed several times and plated on indicated substrates. After a culture period of 16-18 hr, cells were fixed with $4 \%$ (w/v) paraformaldehyde in PBS for $30 \mathrm{~min}$ and washed, and neurite length was determined.

Neurite outgrowth of cerebellar granule cells on transfected $\mathrm{CHO}$ cells was done essentially as described by Tang et al. (1997). In brief, parental and MAG-transfected $\mathrm{CHO}$ cells were used as monolayer underlying dissociated neurons. Cocultures were established by adding $10^{4}$ dissociated cerebellar neurons and maintained for $18 \mathrm{hr}$ in defined medium. For the visualization of neurons grown on monolayers, cells were fixed with $4 \%(\mathrm{w} / \mathrm{v})$ paraformaldehyde in PBS for $15 \mathrm{~min}$, permeabilized with methanol for $10 \mathrm{~min}$, rehydrated in PBS, incubated with anti-GAP-43 (growth-associated protein-43) hybridoma supernatant (clone 10E8; diluted 1:10; gift of Dr. K. Meiri, Syracuse, NY) in PBS containing 3\% (w/v) BSA for $1 \mathrm{hr}$, rinsed with PBS, and incubated with FITCconjugated anti-mouse IgGs (diluted 1:250; Jackson ImmunoResearch, West Grove, PA) in PBS containing 3\% (w/v) BSA for $1 \mathrm{hr}$. Quantification of neurite lengths was performed $16-18 \mathrm{hr}$ after plating on cultures monitored with a Zeiss (Oberkochen, Germany) Axiophot microscope equipped with epifluorescence. Fluorescence pictures were acquired with a 12-bit digital CCD camera (Visicam; Visitron, Puchheim, Germany) and analyzed using MetaMorph software (Universal Imaging Corporation, West Chester, PA). The longest neurite of at least 120 randomly selected individual neurons was measured under each experimental condition for each experiment. Neurite lengths were compared between groups using a one-way ANOVA-test.

$D R G$-oligodendrocyte encounter assay and confocal microscopy. The encounter assay was essentially performed as described previously (Bandtlow et al., 1990; Niederöst et al., 1999). Individual growth cones of dissociated P6 DRG neurons triturated with or without C3 transferase (protocol by Borasio et al., 1989) were viewed by time-lapse video microscopy during their encounter with differentiated oligodendrocytes obtained form P7 rat optic nerves. Briefly, oligodendrocytes were kept in chemically defined medium for $3 \mathrm{~d}$ to allow full differentiation. At this time point, oligodendrocytes are positive for MAG, Nogo-A, and versican V2. Dissociated P6 DRG neurons were added and growth coneoligodendrocyte encounter was observed $6-8 \mathrm{hr}$ after plating. At given time points during growth cone-oligodendrocyte interaction, cultures were fixed by an extraction-fixation procedure (Fritsche et al., 1999) on the microscope stage, and the imaged area was marked at the bottom of the dish for relocalization after immunocytochemistry. To reveal changes in the underlying growth cone cytoskeleton, microtubules were labeled with monoclonal antibody DM1 $\alpha$ directed against $\alpha$-tubulin (Sigma) used at a dilution of 1:400 and F-actin was labeled with rhodamine-conjugated phalloidin (Molecular Probes). Primary antibodies and phalloidin were diluted in blocking solution and applied for 45 min. Dishes were rinsed with $\mathrm{Ca}^{2+}, \mathrm{Mg}^{2+}$ free-PBS to remove unbound antibodies and then soaked for $15 \mathrm{~min}$ in soaking solution. Fluoresceinconjugated secondary antibodies (Jackson ImmunoResearch), at 1:400 dilutions, were applied for $45 \mathrm{~min}$. Immunofluorescence images were 
acquired with a Zeiss LSM 410 inverted laser scanning confocal microscope, using a Zeiss plan-Apochromat $63 \times / 1.4$ numerical aperture oil immersion lens. An Intervall $\mathrm{HeNe}$ laser pretuned to $543 \mathrm{~nm}$ was used for visualizing F-actin, which was tagged with rhodamine, and an argon ion laser pretuned to $488 \mathrm{~nm}$ was used to visualize the fluoresceinlabeled tubulin. A bandpass filter of $590-610 \mathrm{~nm}$ and a dichroic beam splitter of $488 / 543 \mathrm{~nm}$ were selected to obtain the images for doublelabeling experiments. Optical sections $(0.3 \mu \mathrm{m})$ were transferred to a Silicon Graphics (Mountain View, CA) Indigo2 Extreme for processing (Imaris software; Bitplane AG, Zurich, Switzerland).

Rac1 and RhoA activity assays. Measurement of Rac1 and RhoA activities was performed as described previously (Bagrodia et al., 1995; Ren et al., 1999). Cerebellar granule cells from P7 rat pups were grown for $20-24 \mathrm{hr}$ in chemically defined Neurobasal medium (Invitrogen) supplemented with B27 (Invitrogen) (Rac1, $5 \times 10^{6}$ cells; RhoA, $2 \times 10^{7}$ cells) on a poly-L-lysine substrate $(50 \mu \mathrm{g} / \mathrm{ml})$. Cells were treated with MAG-Fc (125 nM), Nogo-66-AP (120 nM), or NiG- $\Delta 20-A P(123 \mathrm{~nm})$ for the indicated time periods. To induce the multimerization of the fusion proteins for optimal receptor activation, $4.2 \mathrm{nM}$ MAG-Fc was preaggregated for $30 \mathrm{~min}$ at room temperature with $50 \mathrm{ng} / \mathrm{ml}$ anti-human Fc (Jackson ImmunoResearch). Nogo-66-AP ( $8 \mathrm{~nm}$ ) or NiG- $\Delta 20-\mathrm{AP}(7 \mathrm{nM})$ were preaggregated with $100 \mathrm{ng} / \mathrm{ml}$ anti-human AP (Sigma). Control cells were incubated with preaggregated $\mathrm{Fc}$ or AP proteins, respectively. After treatment, cells were lysed for 5 min with $400 \mu$ l of the respective ice-cold cell lysis buffer. Cell extract was cleared by centrif ugation $(5 \mathrm{~min}$ at $10,000 \times g$ at $4^{\circ} \mathrm{C}$ ), and $10 \%$ of the total volume was used for assessment of total Rac1 or RhoA content. The remaining lysate was diluted with appropriate binding buffer containing $6 \mu \mathrm{g}$ of GST-CRIB ( $\mathrm{Cdc} 42 / \mathrm{Rac}$ interactive binding protein; kindly provided by Dr. R. Cerione, Cornell University, Ithaca, NY) coupled to glutathione beads for GTP-bound Rac1 or $16 \mu \mathrm{g}$ of GST-Rho-binding domain of mouse rhotekin (GST-RBD; kindly provided by Dr. M. Schwartz, The Scripps Research Institute, La Jolla, CA) coupled to glutathione beads for GTP-bound RhoA. Cell lysates were incubated for $30 \mathrm{~min}$ (Rac1) or 60 min (RhoA) at $4^{\circ} \mathrm{C}$. Beads were then washed three times with binding buffer, and bound material was eluted with $2 \times$ SDS-sample buffer. The total cell lysate and the affinity-precipitated products were resolved by $12.5 \%$ SDS-PAGE and immunoblotted using a mouse monoclonal antiRac1 antibody (1:1000; Transduction Laboratories, Lexington, KY) or a mouse monoclonal anti-RhoA antibody (1:1000; Santa Cruz Biotechnology, Santa Cruz, CA). Peroxidase-conjugated goat anti-mouse IgGs (Sig$\mathrm{ma}$ ) were used as secondary antibodies. Immunoreactive proteins were visualized using an enhanced chemiluminescence detection system (ECL; Amersham Biosciences).

Densitometric analysis were performed using Scion Image software (Scion, Frederick, MD), and the amounts of GTP-bound Rac1 and RhoA were normalized to the total amounts of Rac1 and RhoA in cell lysates, respectively.

Immunocytochemistry. The antibodies used for immunocytochemistry on P7 cerebellar granule cells were 9651 [anti-p75 neurotrophin receptor $\left(\mathrm{p} 75^{\mathrm{NTR}}\right)$ (Huber and Chao, 1995) and anti-NgR (rabbit anti-NgR antisera raised against the three synthetic peptides EQLDLSDNAQLRSVDPA, EVPCSLPQRLAGRDLKR, and GPRRRPGCSRKNRTRS of human NgR and affinity purified by Research Genetics (Carlsbad, CA)]. Cells were fixed with $4 \%$ paraformaldehyde and $5 \%$ sucrose in PBS for $30 \mathrm{~min}$. Primary antibodies were diluted in blocking solution (1:100) and applied for $60 \mathrm{~min}$. Dishes were rinsed with CMF-PBS to remove unbound antibodies, and fluorescein-conjugated anti-rabbit antibodies (Jackson ImmunoResearch), at 1:400 dilutions, were applied for $45 \mathrm{~min}$. Immunofluorescence images were acquired with a Zeiss microscope using a 12-bit digital CCD camera (Visicam; Visitron).

\section{RESULTS}

\section{Involvement of RhoA and ROCK in Nogo-A- and MAG- induced neurite inhibition}

We investigated the possible involvement of small GTP binding molecules of the Rho family in Nogo-A- and MAG-induced neurite inhibition. Purified cerebellar granule cells were grown on substrate-bound recombinant NiG (Nogo-A-specific domain, aa 174-979), on Nogo-66-GST (aa 1026-1091), or on MAG-CHO cells. In agreement with previous studies (Mukhopadhyay et al., 1994; Fournier et al., 2001), we found that all three substrates inhibit neurite outgrowth of P7 cerebellar granule cells (Fig.
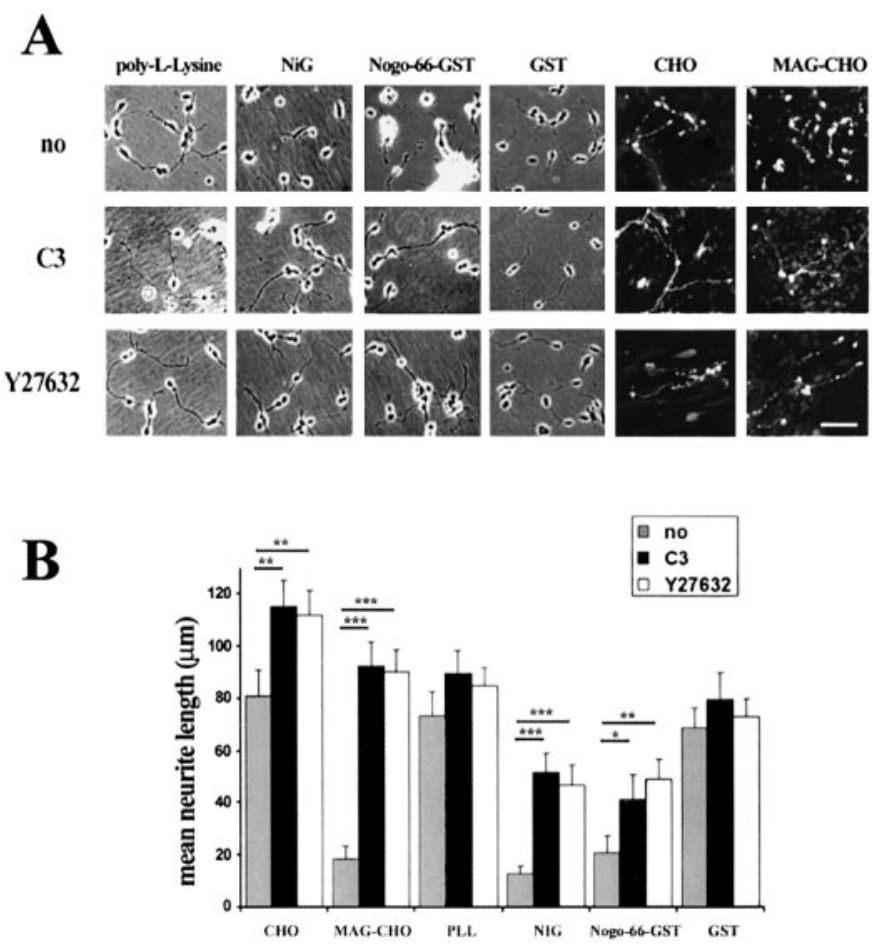

Figure 1. RhoA-dependent inhibition of neurite outgrowth of cerebellar granule cells on Nogo and MAG substrates. Analysis of the inhibitory properties of NiG, Nogo-66-GST, and MAG-CHO. A, Purified cerebellar granule cells of $\mathrm{P} 7$ rat pups were plated on control substrates poly-L-lysine (PLL) $(10 \mu \mathrm{g} / \mathrm{ml})$, GST $(10 \mu \mathrm{g} / \mathrm{ml})$, or CHO control cells and on the inhibitory substrates $\mathrm{NiG}(10 \mu \mathrm{g} / \mathrm{ml})$, Nogo-66-GST $(10 \mu \mathrm{g} / \mathrm{ml})$, and MAG-CHO cells in the absence or presence of C3 transferase or Y27632, respectively. $B$, Neurite length of at least 120 neurons per substrate was measured and presented as the mean neurite length. Note that inhibition of RhoA by C3 transferase or of Rho-kinase by Y27632 restores neurite length on all inhibitory substrates tested. ${ }^{*} p<0.01$; one-way ANOVA. Scale bar, $20 \mu \mathrm{m}$.

$1 A, B)$. Approximately $80 \%$ of the cells had neurites longer than $70 \mu \mathrm{m}$ on control substrates, but only $10-18 \%$ of the cells put out neurites on NiG, Nogo-66-GST, or MAG-CHO. Trituration of granule cells with $20 \mu \mathrm{g} / \mathrm{ml} \mathrm{C} 3$ transferase to inactivate endogenous RhoA activity allowed not only more cells to extend neurites, but their outgrowth response was markedly improved on all three inhibitory substrates. This effect was most evident for cells on an MAG-CHO substrate in which neurite length was restored to untreated control levels, whereas cells grown on NiG or Nogo66-GST substrates showed an improvement of 70 and $60 \%$, respectively (Fig. 1A,B). Previous studies have shown that cell rounding and neurite retraction require the involvement of the RhoA-associated kinase ROCK (Hirose et al., 1998). To determine whether ROCK was involved in the MAG- or Nogo-Ainduced neurite growth inhibition, cerebellar granule cells were treated with various concentrations of a ROCK-selective inhibitor, Y27632 (Ishizaki et al., 2000). Application of Y27632 (15 $\mu \mathrm{M})$ throughout the culture period completely abolished the inhibitory effect of MAG and significantly improved neurite outgrowth response on NiG and Nogo-66-GST substrates (Fig. 1A,B) by 63 and $70 \%$, respectively, compared with untreated control levels. Interestingly, higher concentrations of Y27632 (up to $50 \mu \mathrm{M}$ ) could not further improve the neuronal outgrowth of cerebellar granule cells on NiG or Nogo-66-GST substrates.

These data suggest that RhoA and its downstream effector 
protein ROCK are key regulators mediating Nogo-A- and MAGinduced neurite outgrowth inhibition.

\section{Opposing regulation of RhoA and Rac1 by Nogo-A and MAG}

Many growth factors require dimerization or even oligomerization for optimal receptor binding and activation (Safran et al., 2000). Similarly, binding as well as growth cone collapsing activity of DRG neurons is most effective with dimers or oligomers of MAG or Nogo inhibitory domains (Turnley and Bartlett, 1999; Fournier et al., 2001; Oertle et al., 2001) (Oertle and Schwab, unpublished observations). To directly assess whether Nogo-A and MAG can regulate Rho-GTPase activities, soluble, dimeric fusion proteins of the Nogo inhibitory domains and of the extracellular domain of the MAG molecule were added to cultured cerebellar granule cells, and the amounts of cellular active GTPbound RhoA and Rac1 were measured. As shown in Figure $2 A$, a detailed time course analysis of RhoA activity revealed that equal molar concentrations of MAG-Fc (125 nM), Nogo-66-AP (120 nM), and NiG- $\Delta 20-\mathrm{AP}(123 \mathrm{nM})$, respectively, induced a slow and transient activation of RhoA. Although RhoA activation was maximal at $20 \mathrm{~min}$ for all fusion proteins, their efficacy varied, with Nogo-66-AP being less potent than NiG- $\Delta 20$-AP or MAG-Fc (Fig. $2 A$ ). In contrast to RhoA, the same concentration of fusion proteins induced a slow decrease in the amount of cellular GTP-bound Rac1, reaching minimal levels at $30 \mathrm{~min}$ (Fig. 2A).

To test whether RhoA activation can be further stimulated in response to high-order oligomers, we used MAG-Fc or Nogo-AP fusion proteins that had been preclustered with anti-human-Fc or anti-human-AP antibodies. In these experiments, all preclustered fusion proteins stimulated a very rapid and dramatic increase in RhoA activity with different kinetic profiles (Fig. $2 B$ ). The most prominent effect was seen with preclustered $\mathrm{NiG}-\Delta 20$-AP ( $7 \mathrm{nM}$ ), which peaked within 2-3 min after stimulation and declined within $30 \mathrm{~min}$ to almost basal levels. MAG-Fc (4.2 nM) and Nogo-66-AP (8 nM) were less efficacious, reaching maximal RhoA activity levels at $5 \mathrm{~min}$ and slowly decreasing to basal levels at 30 min after stimulation. Likewise, the amount of cellular GTPbound Rac1 decreased more rapidly than with dimeric fusion proteins, reaching minimal levels at 5-10 min (Fig. 2B), with no apparent recovery to basal levels over a period of $60 \mathrm{~min}$ (data not shown). Interestingly, besides the faster and more dramatic change in Rho activities with oligomerized fusion proteins, less protein was required for maximal stimulation compared with the dimeric fusion proteins. Interestingly, higher concentrations resulted in neither an additional stimulation of RhoA nor a change in time kinetics. No changes were observed using preclustered AP or Fc protein (Fig. 2B) or clustering antibodies (data not shown), excluding the possibility of unspecific effects of the clustering antibodies.

Together, these data not only demonstrate that both Nogo domains and the extracellular domain of MAG exert opposing effects on RhoA and Rac1 activities, i.e., they activate RhoA and suppress Rac1, with different efficacy, but that optimal receptor activation seems to require high-order oligomers.

Next, we evaluated whether the improved neurite outgrowth response of C3 transferase- or Y27632-treated neurons was the result of abolished modulation of Rho GTPase activities by Nogo domains and MAG. C3 transferase effectively suppressed endogenous RhoA activity and specifically inhibited MAG- and Nogoinduced RhoA activation (Fig. 3). Y27632, as expected, did not prevent MAG- and Nogo-induced RhoA activation (Fig. 3) but
A
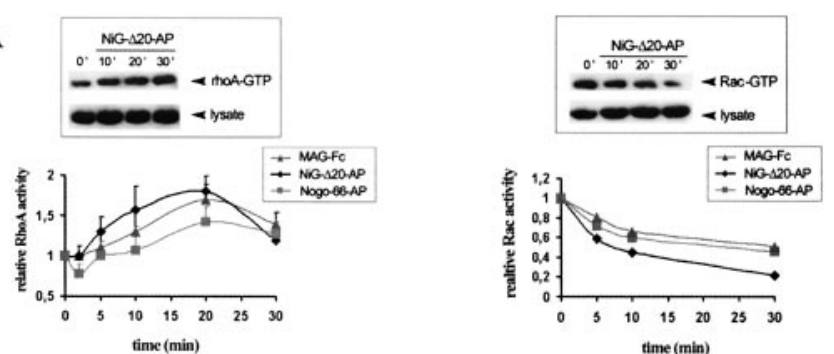

B
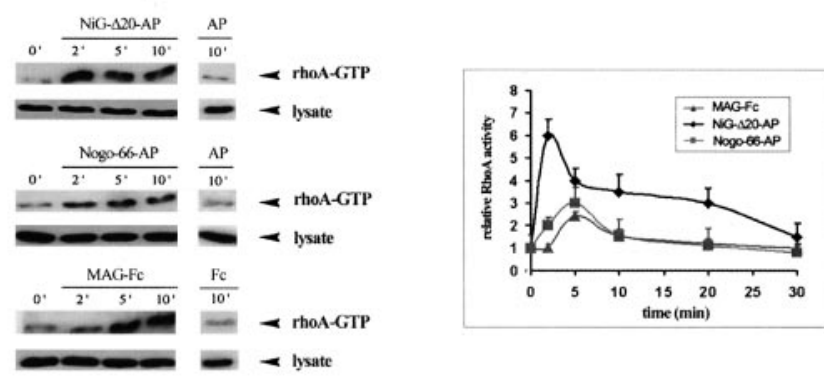

C
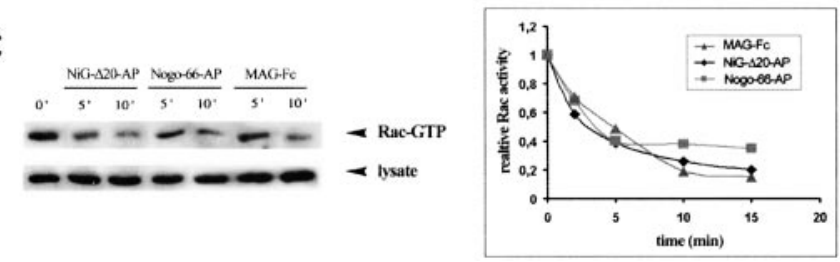

Figure 2. Effects of Nogo-66, NiG- $\Delta 20$, and MAG fusion proteins on RhoA and Rac1 activities in postnatal cerebellar granule cells. Dissociated cerebellar granule cells of P7 rats were grown overnight in chemically defined medium on a poly-L-lysine substrate before stimulation with the growth inhibitory fusion proteins of Nogo-A and MAG. $A$, Changes of RhoA and Rac1 activities over time after the addition of equal molar concentrations (120 nM) of dimeric NiG- $\Delta 20$-AP, Nogo-66-AP, or MAGFc. RhoA and Rac1 activities are indicated by the amount of GST-RBDbound RhoA or GST-CRIB-bound Rac1 normalized to the amount of total RhoA or Rac1 content in the lysates, respectively, as shown for NiG- $\Delta 20$-AP (inset). Values represent RhoA or Rac1 activity and are expressed as fold of the value of cells at time $0 \mathrm{~min}$. Results are means \pm SE from three experiments. Time course of RhoA $(B)$ and Rac1 $(C)$ activities after stimulation with preclustered fusion proteins. Note that less protein is required for maximal stimulation: $7 \mathrm{nM} \mathrm{NiG-} \Delta 20-\mathrm{AP} ; 8 \mathrm{nM}$ Nogo-66-AP; 4.2 nM MAG-Fc. No change is seen with preclustered Fc or AP fusion proteins.

alleviated the inhibitory outgrowth effects of Nogo fragments and MAG (Fig. 1 $A, B$ ). These data suggest that the Y27632 target ROCK acts downstream of RhoA and has no effect in regulating endogenous RhoA activity.

\section{Effect of C3 transferase on DRG neurons encountering differentiated oligodendrocytes}

To assess whether inhibition of endogenous RhoA activity by C3 transferase has not only a protective effect in Nogo- and MAGinduced neurite outgrowth inhibition but also prevents oligodendrocyte-induced growth cone collapse, we monitored interactions of untreated or C3 triturated DRG neurites with oligodendrocytes by time-lapse video microscopy. As shown previously (Bandtlow et al., 1990; Niederöst et al., 1999), growth cone collapse and retraction in untreated cultures was seen in 


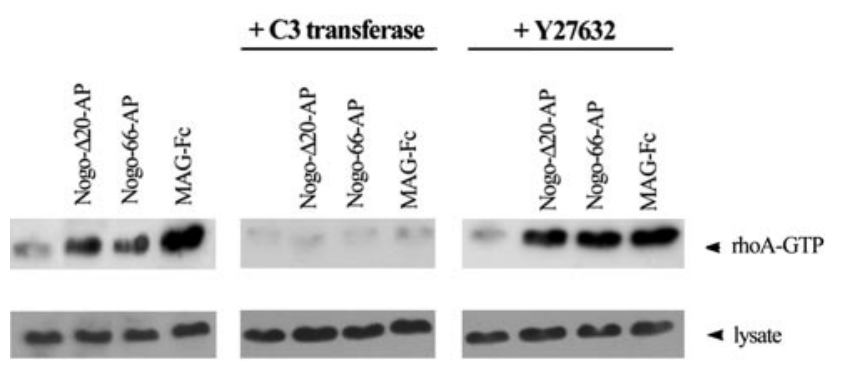

Figure 3. C3 transferase but not Y27632 blocks activation of RhoA. Measurement of RhoA and Rac1 activity of granule cells pretreated with C3 transferase and Y27632, respectively, for $1 \mathrm{hr}$, before stimulation with the preclustered, chimeric fusion proteins for $10 \mathrm{~min}$.

$\sim 80 \%$ of all encounters (Fig. $4 A-D$ ). However, when neurites of C3 triturated DRG cells encountered oligodendrocytes, no contact-mediated growth cone collapse was observed. Instead, growth cones grew over the oligodendrocyte processes without any obvious sign of growth arrest or retraction (Fig. $4 E-H)$. To visualize changes in the organization of actin filaments and microtubules that occur as growth cones collapse, advancing growth cones were monitored by time-lapse video microscopy during their encounter with oligodendrocytes. Cultures were fixed at given time points after contact for immunocytochemical double labeling with rhodamine-phalloidin and anti-tubulin antibodies. In normal advancing growth cones on a laminin substrate, F-actin staining was confined to filopodia and the leading cortical edge of the growth cones with distinct radial striations as shown in Figure $4 A$. Microtubules were found to be tightly bundled and localized in the proximal region of the growth cone. They were often observed in the $\mathrm{C}$-domain of the growth cone as a splayed out structure (Fig. 4A). Contact inhibition of advancing growth cones, however, led to a rapid relocalization of F-actin and to a microtubule reorganization. Ten to $20 \mathrm{~min}$ after firm filopodial contact had been established, F-actin was seen as patches in the central domain of the growth cone (Fig. 4B,C). With the exception of polarized actin fibers confined to filopodia in contact, radial striations had disappeared. Furthermore, microtubules were no longer bundled but seen as defasciculated structures at the neurite shaft (Fig. 4C). Longer time periods of contact induced a significant change of grow th cone shape, characterized by a complete loss of filopodia and a dramatic reduction of growth cone area (Fig. 4D). Complete collapse was distinguished as a club-shaped structure with a thin F-actin-containing tip that was often found in contact with oligodendrocytes or slightly retracted from the original contact site (Fig. 4D). Interestingly, microtubules displayed a complete reorganization, reflected as a loop-like structure within the club-shaped growth cone (Fig. 4D). When neurons were treated with C3 transferase, differences were detected in the staining pattern of rhodamine-phalloidin. F-actin was not distributed following specific patterns (e.g., radial striations) but rather had a diffuse appearance filling the entire growth cone, including its central region in which it appeared in patches (Fig. 4E). Although the overall organization of microtubules of C3 transferase-treated cells was not significantly changed compared with control cells, they seemed to be more bundled in the central domain of the growth cone (Fig. 4E). No apparent cytoskeletal reorganization of F-actin or microtubules was detectable in $\mathrm{C} 3$ transferase-treated growth cones that had advanced onto oligodendrocytes, often found in direct contact with oligodendrocyte processes (Fig. $4 F-H$ ). Together, these results show that inactivation of endogenous RhoA activity by C3 transferase exerts a stabilizing effect on the growth cone cytoskeleton, allowing them to grow on oligodendrocytes.

\section{Involvement of $\mathrm{NgR}$ in Nogo-66-dependent, but not in NiG- or MAG-dependent, effects}

We next investigated whether NgR, a glycan phosphatidyl inositol (GPI)-linked molecule that has been identified recently as a specific receptor for Nogo-66 (Fournier et al., 2001), is involved in mediating the effect of Nogo-66-AP on RhoA activity. Cerebellar granule cells express $\mathrm{NgR}$ as revealed by RT-PCR (data not shown) (Fournier et al., 2001). Neurons were therefore incubated with phosphatidyl inositol-phospholipase C (PI-PLC) $(0.2 \mathrm{U} / \mathrm{ml})$ for $8 \mathrm{hr}$ at $37^{\circ} \mathrm{C}$ to remove GPI-anchored molecules from the cell surface, before Nogo-66-AP was added. As analyzed by immunofluorescence, the enzymatic treatment entirely removed cell surface labeling for $\mathrm{NgR}$ on granule cells but did not prevent the labeling for $\mathrm{p} 75^{\mathrm{NTR}}$, suggesting that the enzymatic treatment had no effect on integral plasma membrane proteins (Fig. 5A). Whereas PI-PLC-treated cells were no longer inhibited on a GST-Nogo-66 substrate, neurite outgrowth of the same cells was still strongly impaired on $\mathrm{NiG}$ and MAG-CHO cells (Fig. 5B). In addition, RhoA activity pull-down assays revealed that Nogo-66AP-induced RhoA activation was prevented after PI-PLC treatment, but the increase of GTP-bound RhoA by preclustered NiG- $\Delta 20$-AP or MAG-Fc fusion proteins was not significantly changed (Fig. 5C). These results not only indicate that $\mathrm{NgR}$ is necessary to mediate the responses to Nogo-66 but that NiG as well as MAG are likely to act on different cell surface binding proteins or receptors to induce RhoA activation.

\section{DISCUSSION}

We studied the involvement of small GTPases of the Rho family in mediating the responses of the Nogo-A-specific domain NiG, Nogo-66, and MAG. Both RhoA and Rac1 were found to be regulated in an antagonistic manner by all three neurite growthinhibitory components. Inhibition of the RhoA-ROCK pathway greatly improved neurite outgrowth response of cerebellar granule cells. C3 transferase treatment abolished RhoA activation and rendered growth cones of dorsal root ganglion cells less responsive to oligodendrocyte contact-induced collapse. Furthermore, neuronal NgR, known to bind Nogo-66 and MAG, is necessary for Nogo-66-induced RhoA activation but not for mediating effects of MAG or NiG. Altogether, this study provides compelling evidence for a key role of Rho GTPases in the cytosolic mechanisms induced by Nogo-A and MAG.

\section{Rho family members as mediators of neurite inhibition}

Previous observations implied the requirement of the RhoAROCK pathway for neurite extension on myelin inhibitory test substrates (Lehmann et al., 1999; Vastrik et al., 1999; Vinson et al., 2001); however, they did not address whether components of CNS myelin, such as Nogo-A and MAG, can instructively regulate the activities of Rho GTPases. Here we demonstrate that, in cultured P7 cerebellar granule cells, the effect of Nogo-A and MAG are mediated by common pathways, resulting in the activation of RhoA and concomitant inactivation of Rac1. Inhibition of RhoA and of its downstream-effector protein Rho-kinase ROCK almost completely abolishes MAG activity but alleviates inhibition by Nogo-66 or NiG only partly by $60-70 \%$. Our own data suggest that Nogo-A may induce additional pathways to exert its full activity. First, no significant RhoA activation could be measured in C3 transferase-loaded cells (Fig. 3), implying that 


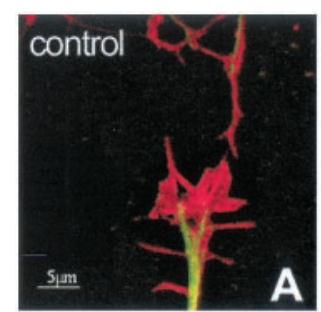

pre

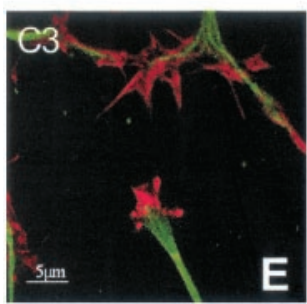

pre

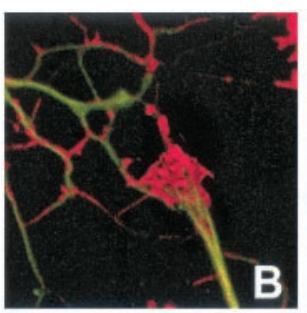

$10 \mathrm{~min}$

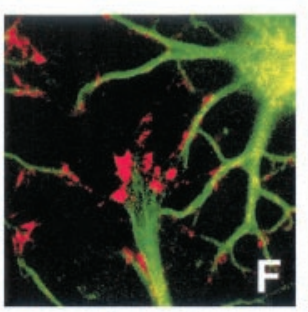

$10 \mathrm{~min}$

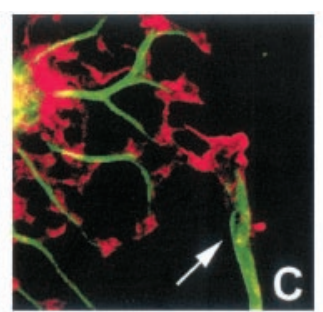

$15 \mathrm{~min}$

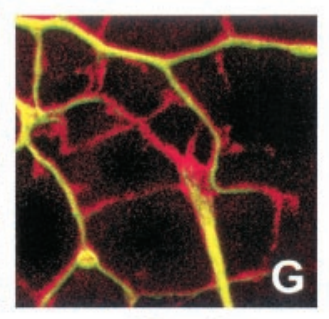

20 min

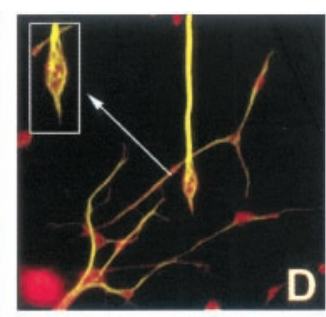

$60 \mathrm{~min}$

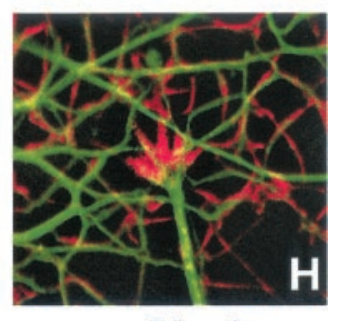

$50 \mathrm{~min}$

Figure 4. Confocal images showing the change in organization of actin filaments and microtubules in growth cones on encounter of oligodendrocytes. Dissociated rat DRG neurons were triturated with either buffer or C3 transferase $(30 \mu \mathrm{g} / \mathrm{ml})$ and cocultured with differentiated oligodendrocytes. Encounter of growth cones were monitored with time-lapse video microscopy for indicated time periods, and cultures were fixed for immunostaining with antibodies against actin filaments (red) and $\alpha$-tubulin (green) as described in Materials and Methods. $A-D$, Buffer triturated growth cones before contact $(A), 10$ and $15 \mathrm{~min}$ after first filopodial contact $(B, C)$, and after $60 \mathrm{~min}$ in contact with oligodendrocyte processes (D). $E-H$, C3 transferase triturated growth cones before contact $(E), 10-20$ $\min (F, G)$, and $50 \mathrm{~min}(H)$ after initial contact. Note that, under control situations $(A-D)$, oligodendrocyte contact leads to a rapid arrest $(B)$ and subsequent collapse of the growth cone structure $(C, D)$, accompanied by a loss of

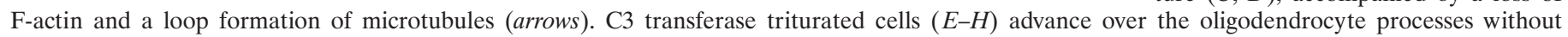
apparent signs of arrest or collapse. Scale bars, $5 \mu \mathrm{m}$

the concentration used to inhibit RhoA was sufficient. Second, considerably higher concentrations of the ROCK inhibitor Y27632 (up to $50 \mu \mathrm{M}$; $\mathrm{EC}_{50}$ for most neuronal cells is $10 \mu \mathrm{M}$ ) did not further improve the neurite outgrowth response of cerebellar granule cells on Nogo-66-GST and NiG substrates.

Soluble recombinant Nogo-66 or eukaryotically expressed Nogo-A-Fc and MAG-Fc at concentrations of $\sim 100 \mathrm{~nm}$ potently inhibit neurite growth of cerebellar granule cells (DeBellard et al., 1996; Tang et al., 1997; GrandPré et al., 2000; Prinjha et al., 2000; Fournier et al., 2001). The same concentrations actively modulate Rho activities (Fig. 2), with slight differences in their efficacy and kinetic profiles. Antibody-mediated oligomerization of the inhibitory fusion proteins induce a more rapid and dramatic modulation of Rho activities than dimeric proteins. Because in our hands preclustering also results in improved ligand binding properties and growth cone-collapsing activities (Oertle and Schwab, unpublished observations), we conclude that optimal receptor activation seems to be facilitated by high-order oligomers of both MAG and Nogo-A.

\section{MAG and Nogo-A induce antagonistic effects of Rho GTPases}

Mutually antagonistic effects of RhoA and Rac/Cdc42 have been observed for other axonal guidance cues. EphrinA5, a repulsive guidance molecule important for establishing the anteroposterior retinotectal topographic map, was shown to activate RhoA and inhibit Rac1 (Wahl et al., 2000). Furthermore, exposure of retinal ganglion cells or cortical neurons to ephrin A1 stimulated activation of Eph4 receptor, which associates with ephexin, a novel Rho GTPase GEF (guanine exchange factor). Subsequently, ephexin looses its ability to activate Rac1 and $\mathrm{Cdc} 42$, becoming a specific RhoA GEF and, in this way, is mediating growth cone collapse (Shamah et al., 2001). Sema3A, a member of the semaphorin family of guidance molecules, also signals through the Rho GTPases and requires the functions of both Rac and RhoA (Jin and Strittmatter, 1997; Driessens et al., 2001; Liu and Strittmatter, 2001). In contrast, Netrin, when acting as a chemoattractant, was shown to induce Rac1 and to reduce RhoA activities (Li et al., 2002). Together with these and other studies, our data support a current, although rather simplified, model that suggests that attractive guidance cues inhibit RhoA but activate Rac1 or Cdc42 to promote directed axonal growth, whereas repulsive cues inhibit Rac1 and/or Cdc42 and stimulate RhoA to induce neurite retraction (Dickson, 2001).

\section{Cross-talk between Rho GTPases}

Cross-talk between RhoA and Rac-Cdc42 pathways may occur at several levels. Active RhoA is known to signal to its effector protein ROCK, which phosphorylates myosin light chain (MLC) phosphatase (Kimura et al., 1996), as well as MLC itself (Amano et al., 1996). This increase in MLC phosphorylation consequently increases actomyosin-based contractility. Active Rac, on the other hand, signals to its effector protein PAK1, a serine-threonine kinase (Manser et al., 1995) that inhibits MLC kinase, resulting in decreased phosphorylation of myosin light chain and hence decreased actomyosin contractility (Sanders et al., 1999). Thus, RhoA and Rac have antagonistic effects on myosin contractility. This is consistent with the opposing modulation of both pathways that underlie Nogo-A- and MAG-induced effects.

The downregulation of Rac1 activity may also affect the Rac effector PI4P5K (phosphatidylinositol-4-phosphate 5-kinase), which generates phosphatidylinositol-4,5-bisphosphate (PIP2) (Tolias et al., 1995, 1998; Carpenter et al., 1999). PIP2 has been shown to bind to the N-WASP (neural Wiskott Aldrich syndrome protein) control region to stimulate actin assembly (Rohatgi et al., 2000). PIP2 also promotes the extension of existing filaments by inhibiting barbed end capping proteins (Janmey and Stossel, 1987). These pathways are likely to be critical for the forward extension of growth cones, as suggested by the recent finding that a dominant-negative form of N-WASP inhibits neurite extension in primary hippocampal neurons (Banzai et al., 2000). Thus, Nogo-A- and MAG-induced inactivation of Rac may lead to a subsequent decrease in active N-WASP and in PIP2 production 
A
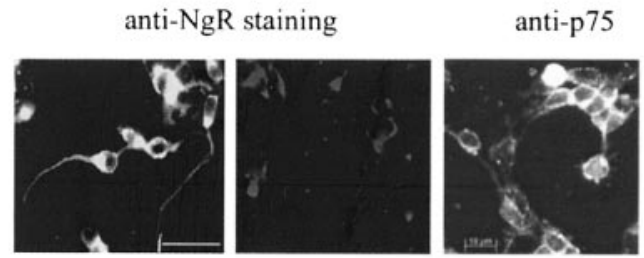

PI-PLC $+$

$+$

B

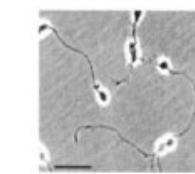

PLL/LN PI-PLC: +

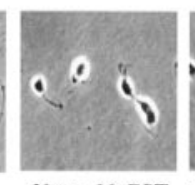

Nogo-66-GST Nogo-66-GST

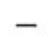

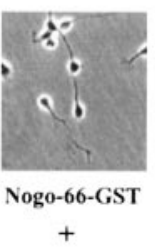

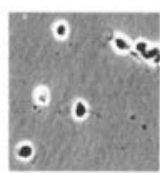

$\mathrm{NiG}$

$+$

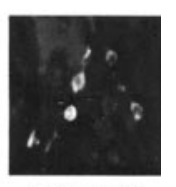

MAG-CHO

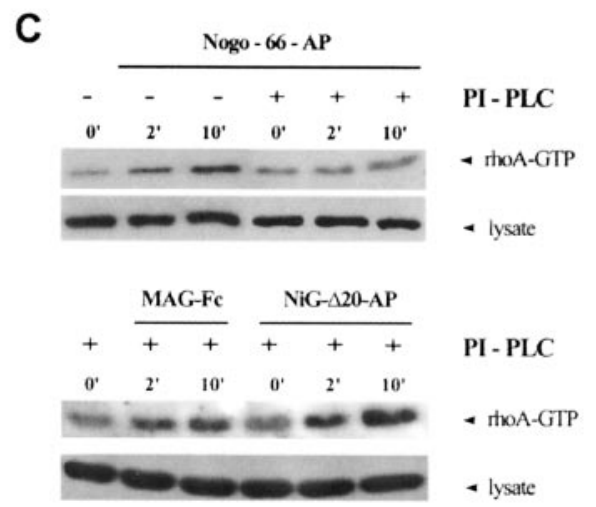

Figure 5. $\mathrm{NgR}$ is only required for Nogo-66-mediated, but not for MAGor NiG-mediated, effects. $A$, Immunocytochemical detection of $\mathrm{NgR}$ on purified cerebellar granule cells of P7 rat pups $18 \mathrm{hr}$ after plating on a poly-L-lysine substrate before and after PI-PLC treatment. Note that $\mathrm{NgR}$ but not $75^{\mathrm{NTR}}$ immunolabeling is abolished by PI-PLC treatment. $B$, Removal of NgR by PI-PLC abolishes neurite inhibition on GST-Nogo-66 but not on NiG or MAG; $C$, PI-PLC prevents induction of RhoA activation by Nogo-66-AP but not by NiG- $\Delta 20-A P$ or MAG-Fc. Scale bar, 20 $\mu \mathrm{m} . P L L / L N$, Poly-L-lysine/laminin.

and arrest growth cone motility by shutting down the local supply of monomeric actin.

\section{Cytoskeletal changes in contact-mediated growth cone collapse}

Recent studies have demonstrated that LIM kinase is not only a target of ROCK (Maekawa et al., 1999) but also of PAK1 (Yang et al., 1998; Maekawa et al., 1999). LIM kinase phosphorylates and thereby inactivates the actin-depolymerizing protein cofilin (Arber et al., 1998; Yang et al., 1998). Such a LIM kinasedependent phosphorylation of cofilin has been shown to be required in Sema3A-induced growth cone collapse of DRG neurons (Aizawa et al., 2001). Because cofilin is important for turnover of actin filaments (Moon and Drubin, 1995; Carlier et al., 1997), its inactivation may initially stabilize existing actin filaments. However, because cofilin phosphorylation was seen only transiently, growth cone collapse may occur because of the presence of a phosphatase that counteracts the actions of LIM kinase and restores the actin-depolymerizing effects of cofilin in a continuous manner (Meberg et al., 1998; Aizawa et al., 2001; Niwa et al., 2002). A similar mechanism may account for the contact-mediated growth cone collapse in oligodendrocyte encounters described in the present study. Filopodial contacts must activate distinct biochemical pathways at the filopodial tip, which differentially modulate the molecular machinery within the growth cone. Although the precise mechanism(s) involved in the spatial and temporal regulation of actin filament polymerizationdepolymerization during contact-mediated growth cone collapse is not known, altering the coordinated assembly and disassembly of actin filaments via Rho GTPases could contribute to collapse. Thus, Nogo-A- and MAG-mediated filopodial contact may lead to the activation and recruitment of RhoA and ROCK, which may initially facilitate filopodial stabilization by localized cofilin phosphorylation via LIM kinase. The subsequent activation of a phosphatase to maintain cofilin actin-depolymerizing activity, together with the ROCK-induced contractility of the cortical actin-myosin system, would explain the subsequent complete collapse of the growth cone structure.

\section{Role of NgR in mediating Nogo-A and MAG effects}

Recently, NgR, the GPI-linked receptor for Nogo-66 (Fournier et al., 2001), has been identified to act as a binding molecule for other inhibitory molecules, such as MAG (Domeniconi et al., 2002; Liu et al., 2002) and oligodendrocyte-myelin glycoprotein (Wang et al., 2002). Our own studies suggest that NgR is necessary for Nogo-66-induced RhoA activation, although they do not resolve the question whether $\mathrm{NgR}$ is the sole receptor for Nogo-66 or whether it acts in conjunction with other molecules to form a receptor complex leading to RhoA activation. Our data, however, do not support the recent finding that $\mathrm{NgR}$ is required for MAG-mediated neurite inhibition (Domeniconi et al., 2002; Liu et al., 2002). In our hands, neither NgR nor other GPI-linked proteins seem to be necessary for cerebellar granule cells to be inhibited on MAG-CHO or NiG substrates, nor are GPI-linked proteins required for the regulation of Rho GTPases by MAG-Fc or NiG- $\Delta 20-\mathrm{AP}$. Our data do not exclude that $\mathrm{NgR}$ can bind MAG (Domeniconi et al., 2002; Liu et al., 2002), but they suggest that MAG as well as NiG can act on other neuronal binding molecules or receptors to impart their effects. These data are in line with the recent finding that NiG can inhibit neurite outgrowth of cells that lack NgR expression (Oertle and Schwab, unpublished observations), but the identity of the receptor specific for NiG remains still unknown. A likely candidate for a different functional MAG receptor is the low-affinity $\mathrm{p} 75^{\mathrm{NTR}}$. Recent results show that MAG cannot only bind to $\mathrm{p} 75^{\mathrm{NTR}}$ but that $\mathrm{p} 75^{\mathrm{NTR}}$ is required to act as a functional receptor to convey MAG-induced RhoA activation (Yamashita et al., 2002). Because cerebellar granule cells express high levels of $\mathrm{p} 75^{\mathrm{NTR}}$ (Yamashita et al., 2002; this study), it is possible that $\mathrm{p} 75^{\mathrm{NTR}}$ on its own is sufficient to mediate MAG-induced RhoA activation in the absence of $\mathrm{NgR}$.

Together, our studies demonstrate that Rho GTPases are key signaling molecules mediating Nogo-A- and MAG-induced growth inhibition. Although much is yet to be learned about the temporal and spatial relationships between GTPase activities and the subsequent changes in cytoskeletal organization, our studies will help to elucidate the molecular mechanisms involved in preventing axonal growth in the CNS. 


\section{REFERENCES}

Aizawa H, Wakatsuki S, Ishii A, Moriyama K, Sasaki Y, Ohashi K, Sekine-Aizawa Y, Sehara-Fujisawa A, Mizuno K, Goshima Y, Yahara I (2001) Phosphorylation of cofilin by LIM-kinase is necessary for semaphorin 3A- induced growth cone collapse. Nat Neurosci 4:367-373.

Amano M, Ito M, Kimura K, Fukata Y, Chihara K, Nakano T, Matsuura Y, Kaibuchi K (1996) Phosphorylation and activation of myosin by Rho-associated kinase (Rho-kinase). J Biol Chem 271:20246-20249.

Arber S, Barbayannis FA, Hanser H, Schneider C, Stanyon CA, Bernard O, Caroni P (1998) Regulation of actin dynamics through phosphorylation of cofilin by LIM- kinase. Nature 393:805-809.

Bagrodia S, Derijard B, Davis RJ, Cerione RA (1995) Cdc42 and PAKmediated signaling leads to Jun kinase and p38 mitogen- activated protein kinase activation. J Biol Chem 270:27995-27998.

Bandtlow C, Zachleder T, Schwab ME (1990) Oligodendrocytes arrest neurite growth by contact inhibition. J Neurosci 10:3837-3848.

Banzai Y, Miki H, Yamaguchi H, Takenawa T (2000) Essential role of neural Wiskott-Aldrich syndrome protein in neurite extension in PC12 cells and rat hippocampal primary culture cells. J Biol Chem 275:11987-11992.

Bishop AL, Hall A (2000) Rho GTPases and their effector proteins. Biochem J 348:241-255.

Bito H, Furuyashiki T, Ishihara H, Shibasaki Y, Ohashi K, Mizuno K, Maekawa M, Ishizaki T, Narumiya S (2000) A critical role for a Rho-associated kinase, p160ROCK, in determining axon outgrowth in mammalian CNS neurons. Neuron 26:431-441.

Borasio GD, John J, Wittinghofer A, Barde YA, Sendtner M, Heumann $\mathrm{R}$ (1989) ras p21 protein promotes survival and fiber outgrowth of cultured embryonic neurons. Neuron 2:1087-1096.

Carlier MF, Laurent V, Santolini J, Melki R, Didry D, Xia GX, Hong Y, Chua NH, Pantaloni D (1997) Actin depolymerizing factor (ADF/ cofilin) enhances the rate of filament turnover: implication in actinbased motility. J Cell Biol 136:1307-1322.

Carpenter CL, Tolias KF, Van Vugt A, Hartwig J (1999) Lipid kinases are novel effectors of the GTPase Rac1. Adv Enzyme Regul 39:299-312.

Chen MS, Huber AB, van der Haar ME, Frank M, Schnell L, Spillmann AA, Christ F, Schwab ME (2000) Nogo-A is a myelin-associated neurite outgrowth inhibitor and an antigen for monoclonal antibody IN-1. Nature 403:434-439.

DeBellard ME, Tang S, Mukhopadhyay G, Shen YJ, Filbin MT (1996) Myelin-associated glycoprotein inhibits axonal regeneration from a variety of neurons via interaction with a sialoglycoprotein. Mol Cell Neurosci 7:89-101.

Dickson BJ (2001) Rho GTPases in growth cone guidance. Curr Opin Neurobiol 11:103-110.

Domeniconi M, Cao Z, Spencer T, Sivasankaran R, Wang K, Nikulina E, Kimura N, Cai H, Deng K, Gao Y, He Z, Filbin M (2002) Myelinassociated glycoprotein interacts with the Nogo66 receptor to inhibit neurite outgrowth. Neuron 35:283-290.

Driessens MH, Hu H, Nobes CD, Self A, Jordens I, Goodman CS, Hall A (2001) Plexin-B semaphorin receptors interact directly with active Rac and regulate the actin cytoskeleton by activating Rho. Curr Biol 11:339-344.

Flanagan JG, Leder P (1990) The kit ligand: a cell surface molecule altered in steel mutant fibroblasts. Cell 63:185-194.

Fournier AE, GrandPre T, Strittmatter SM (2001) Identification of a receptor mediating Nogo-66 inhibition of axonal regeneration. Nature 409:341-346.

Fritsche J, Reber BF, Schindelholz B, Bandtlow CE (1999) Differential cytoskeletal changes during growth cone collapse in response to hSema III and thrombin. Mol Cell Neurosci 14:398-418.

GrandPré T, Nakamura F, Vartanian T, Strittmatter SM (2000) Identification of the Nogo inhibitor of axon regeneration as a Reticulon protein. Nature 403:439-444.

Hatten ME (1985) Neuronal regulation of astroglial morphology and proliferation in vitro. J Cell Biol 100:384-396.

Hirose M, Ishizaki T, Watanabe N, Uehata M, Kranenburg O, Moolenaar WH, Matsumura F, Maekawa M, Bito H, Narumiya S (1998) Molecular dissection of the Rho-associated protein kinase (p160ROCK)regulated neurite remodeling in neuroblastoma N1E-115 cells. J Cell Biol 141:1625-1636.

Huber AB, Schwab ME (2000) Nogo-A, a potent inhibitor of neurite outgrowth and regeneration. Biol Chem 381:407-419.

Huber LJ, Chao MV (1995) Mesenchymal and neuronal cell expression of the p75 neurotrophin receptor gene occur by different mechanisms. Dev Biol 167:227-238.

Ishizaki T, Uehata M, Tamechika I, Keel J, Nonomura K, Maekawa M, Narumiya S (2000) Pharmacological properties of Y-27632, a specific inhibitor of rho-associated kinases. Mol Pharmacol 57:976-983.
Janmey PA, Stossel TP (1987) Modulation of gelsolin function by phosphatidylinositol 4, 5-bisphosphate. Nature 325:362-364.

Jin Z, Strittmatter SM (1997) Rac1 mediates collapsin-1-induced growth cone collapse. J Neurosci 17:6256-6263.

Kimura K, Ito M, Amano M, Chihara K, Fukata Y, Nakafuku M, Yamamori B, Feng J, Nakano T, Okawa K, Iwamatsu A, Kaibuchi K (1996) Regulation of myosin phosphatase by Rho and Rho-associated kinase (Rho-kinase). Science 273:245-248.

Lehmann M, Fournier A, Selles-Navarro I, Dergham P, Sebok A, Leclerc N, Tigyi G, McKerracher L (1999) Inactivation of Rho signaling pathway promotes CNS axon regeneration. J Neurosci 19:7537-7547.

Li M, Shibata A, Li C, Braun PE, McKerracher L, Roder J, Kater SB, David S (1996) Myelin-associated glycoprotein inhibits neurite/ axon growth and causes growth cone collapse. J Neurosci Res $46: 404-414$

Li X, Saint-Cyr-Proulx E, Aktories K, Lamarche-Vane N (2002) Rac1 and $\mathrm{Cdc} 42$ but not RhoA or Rho-kinase activities are required for neurite outgrowth induced by the Netrin-1 receptor DCC (deleted in colorectal cancer) in N1E-115 neuroblastoma cells. J Biol Chem 277:15207-15214.

Liu BP, Strittmatter SM (2001) Semaphorin-mediated axonal guidance via Rho-related G proteins. Curr Opin Cell Biol 13:619-626.

Liu BP, Fournier A, GrandPre T, Strittmatter SM (2002) Myelinassociated glycoprotein as a functional ligand for the Nogo-66 receptor. Science 297:1190-1193.

Luo L (2000) Rho GTPases in neuronal morphogenesis. Nat Rev Neurosci 1:173-180.

Maekawa M, Ishizaki T, Boku S, Watanabe N, Fujita A, Iwamatsu A, Obinata T, Ohashi K, Mizuno K, Narumiya S (1999) Signaling from Rho to the actin cytoskeleton through protein kinases ROCK and LIM-kinase. Science 285:895-898.

Manser E, Chong C, Zhao ZS, Leung T, Michael G, Hall C, Lim L (1995) Molecular cloning of a new member of the p21-Cdc42/Racactivated kinase (PAK) family. J Biol Chem 270:25070-25078.

Meberg PJ, Ono S, Minamide LS, Takahashi M, Bamburg JR (1998) Actin depolymerizing factor and cofilin phosphorylation dynamics: response to signals that regulate neurite extension. Cell Motil Cytoskeleton 39:172-190.

Moon A, Drubin DG (1995) The ADF/cofilin proteins: stimulusresponsive modulators of actin dynamics. Mol Biol Cell 6:1423-1431.

Mukhopadhyay G, Doherty P, Walsh FS, Crocker PR, Filbin MT (1994) A novel role for myelin-associated glycoprotein as an inhibitor of axonal regeneration. Neuron 13:757-767.

Niederöst BP, Zimmermann DR, Schwab ME, Bandtlow CE. Bovine (1999) CNS myelin contains neurite growth-inhibitory activity associated with chondroitin sulfate proteoglycans. J Neurosci 19:8979-8989.

Niwa R, Nagata-Ohashi K, Takeichi M, Mizuno K, Uemura T (2002) Control of actin reorganization by Slingshot, a family of phosphatases that dephosphorylate ADF/cofilin. Cell 108:233-246.

Oertle T, Gillieron O, Bandtlow CE, Schwab ME (2001) Molecular and genomic characterization of the Nogo/Reticulon family of proteins. Soc Neurosci Abstr 27:671.

Prinjha R, Moore SE, Vinson M, Blake S, Morrow R, Christie G, Michalovich D, Simmons DL, Walsh FS (2000) Inhibitor of neurite outgrowth in humans. Nature 403:383-384.

Qiu J, Cai D, Filbin MT (2000) Glial inhibition of nerve regeneration in the mature mammalian CNS. Glia 29:166-174.

Ren XD, Kiosses WB, Schwartz MA (1999) Regulation of the small GTP-binding protein Rho by cell adhesion and the cytoskeleton. EMBO J 18:578-585.

Rohatgi R, Ho HY, Kirschner MW (2000) Mechanism of N-WASP activation by CDC42 and phosphatidylinositol 4, 5- bisphosphate. J Cell Biol 150:1299-1310.

Safran M, Eisenstein M, Aviezer D, Yayon A (2000) Oligomerization reduces heparin affinity but enhances receptor binding of fibroblast growth factor 2. Biochem J 345:107-113.

Sanders LC, Matsumura F, Bokoch GM, de Lanerolle P (1999) Inhibition of myosin light chain kinase by p21-activated kinase. Science 283:2083-2085

Schafer M, Fruttiger M, Montag D, Schachner M, Martini R (1996) Disruption of the gene for the myelin-associated glycoprotein improves axonal regrowth along myelin in $\mathrm{C} 57 \mathrm{BL} / \mathrm{Wlds}$ mice. Neuron 16:1107-1113.

Shamah SM, Lin MZ, Goldberg JL, Estrach S, Sahin M, Hu L, Bazalakova M, Neve RL, Corfas G, Debant A, Greenberg ME (2001) EphA receptors regulate growth cone dynamics through the novel guanine nucleotide exchange factor ephexin. Cell 105:233-244.

Tang S, Woodhall RW, Shen YJ, DeBellard ME, Saffell JL, Doherty P, Walsh FS, Filbin MT (1997) Soluble myelin-associated glycoprotein (MAG) found in vivo inhibits axonal regeneration. Mol Cell Neurosci 9:333-346.

Tang S, Qiu J, Nikulina E, Filbin MT (2001) Soluble myelin-associated 
glycoprotein released from damaged white matter inhibits axonal regeneration. Mol Cell Neurosci 18:259-269.

Tolias KF, Cantley LC, Carpenter CL (1995) Rho family GTPases bind to phosphoinositide kinases. J Biol Chem 270:17656-17659.

Tolias KF, Rameh LE, Ishihara H, Shibasaki Y, Chen J, Prestwich GD, Cantley LC, Carpenter CL (1998) Type I phosphatidylinositol-4phosphate 5-kinases synthesize the novel lipids phosphatidylinositol 3, 5-bisphosphate and phosphatidylinositol 5-phosphate. J Biol Chem 273:18040-18046.

Turnley AM, Bartlett PF (1999) Nerve growth factor modulates myelinassociated glycoprotein binding to sensory neurons. Int J Dev Neurosci 17:109-119.

Vastrik I, Eickholt BJ, Walsh FS, Ridley A, Doherty P (1999) Sema3Ainduced growth-cone collapse is mediated by Rac1 amino acids 17-32. Curr Biol 9:991-998.

Vinson M, Strijbos PJ, Rowles A, Facci L, Moore SE, Simmons DL,
Walsh FS. Myelin-associated glycoprotein interacts with ganglioside GT1b (2001) A mechanism for neurite outgrowth inhibition. J Biol Chem 276:20280-20285.

Wahl S, Barth H, Ciossek T, Aktories K, Mueller BK (2000) Ephrin-A5 induces collapse of growth cones by activating Rho and Rho kinase. J Cell Biol 149:263-270.

Wang KC, Koprivica V, Kim JA, Sivasankaran R, Guo Y, Neve RL, He Z (2002) Oligodendrocyte-myelin glycoprotein is a Nogo receptor ligand that inhibits neurite outgrowth. Nature 417:941-944.

Yamashita T, Higuchi H, Tohyama M (2002) The p75 receptor transduces the signal from myelin-associated glycoprotein to Rho. J Cell Biol 157:565-570.

Yang N, Higuchi O, Ohashi K, Nagata K, Wada A, Kangawa K, Nishida E, Mizuno K (1998) Cofilin phosphorylation by LIM-kinase 1 and its role in Rac-mediated actin reorganization. Nature 393:809-812. 